\title{
Accelerating Polaritons with External Electric and Magnetic Fields
}

\author{
T. Chervy $\odot,{ }^{1, *}$ P. Knüppel $\odot,{ }^{1, *}$ H. Abbaspour $\odot,{ }^{1}$ M. Lupatini, ${ }^{2}$ S. Fält $\odot,{ }^{1,2}$ \\ W. Wegscheider, ${ }^{2}$ M. Kroner, ${ }^{1}$ and A. Imamoğlu ${ }^{1}$ \\ ${ }^{1}$ Institute of Quantum Electroncis, ETH Zürich, CH-8093 Zürich, Switzerland \\ ${ }^{2}$ Solid State Physics Laboratory, ETH Zürich, CH-8093 Zürich, Switzerland
}

(Received 15 November 2019; accepted 14 January 2020; published 19 February 2020)

\begin{abstract}
It is widely assumed that photons cannot be manipulated using electric or magnetic fields. Even though hybridization of photons with electronic polarization to form exciton-polaritons has paved the way to a number of groundbreaking experiments in semiconductor microcavities, the neutral bosonic nature of these quasiparticles has severely limited their response to external gauge fields. Here, we demonstrate polariton acceleration by external electric and magnetic fields in the presence of nonperturbative coupling between polaritons and itinerant electrons, leading to formation of new quasiparticles termed polaron-polaritons. We identify the generation of electron density gradients by the applied fields to be primarily responsible for inducing a gradient in polariton energy, which in turn leads to acceleration along a direction determined by the applied fields. Remarkably, we also observe that different polarization components of the polaritons can be accelerated in opposite directions when the electrons are in $\nu=1$ integer quantum Hall state.
\end{abstract}

DOI: 10.1103/PhysRevX.10.011040

Controlling photons with external electric or magnetic fields is an outstanding goal. On the one hand, coupling photons to artificial gauge fields holds promises for the realization of topological and strongly correlated phases of light [1-4]. On the other hand, effecting forces on photons constitutes both a problem of fundamental interest in electromagnetism and an important step in view of technological applications [5-8]. One promising avenue toward this goal is to hybridize photons with material excitations that are genuinely sensitive to gauge fields [9]. In this nonperturbative regime, exciton-polariton states are formed, ensuring that the forces acting on the material excitations are directly imprinted onto the photon. However, the neutral bosonic nature of polaritons has so far severely limited their response to gauge fields [10-13].

A particularly appealing approach to circumvent this limitation is to leverage on the interaction between excitons and charges. Indeed, early reports on the drift of trions in an electric field [14,15], as well as on the Coulomb drag effect in bilayer systems [16-19], indicated that it may be possible to manipulate neutral excitations using electric fields in a solid-state setting. Recently, experimental [20] and theoretical [21] studies reported the electrical control of

\footnotetext{
*These authors contributed equally to this work.
}

Published by the American Physical Society under the terms of the Creative Commons Attribution 4.0 International license. Further distribution of this work must maintain attribution to the author(s) and the published article's title, journal citation, and DOI.
Subject Areas: Condensed Matter Physics, Optics, Photonics

the speed of a polariton superfluid, raising new questions and possibilities regarding the interplay between the normal and condensed fractions of the fluid in the presence of electron-exciton interactions.

While interactions between polaritons and electrons have been proposed and analyzed as a mechanism for polariton thermalization [22-25], early studies reported the modifications to polariton resonances in the presence of a Fermi sea [26-28]. These modifications stem from dispersive interactions between the polarizable excitonic component of the polariton and charge-density fluctuations of the Fermi sea [29-31]. In a typical setting where excitons are hosted by a GaAs quantum well (QW), the existence of a spin-singlet trion bound channel leads to the formation of two optically active branches referred to as attractive and repulsive polarons $[32,33]$. These polaronic states consist of an exciton dressed by collective trion-hole excitations of the Fermi sea, where the spin of the photoexcited electron is opposite to that of the dressing Fermi sea electrons [32-34]. Upon decreasing the electron density, the quasiparticle weight of the repulsive polaron branch, quantifying its excitonic character, increases. In the limit of vanishing electron density, the repulsive (attractive) polaron asymptotically becomes the bare exciton (molecular trion) state with strong (vanishing) coupling to the cavity mode [32]. The many-body polaronic states are expected to be charge neutral [31,32], suggesting the absence of coupling to external fields. In a recent theoretical study, however, it was shown that neutral polarons are sensitive to the average force on electrons, leading to a finite polaron 
transconductivity in the nonequilibrium limit—an effect that should be observable even when polarons hybridize with cavity photons [35].

In the present work, we demonstrate experimentally that external electric and magnetic fields effect forces on polaronpolaritons. In contrast to earlier proposals, we find that the observed polariton acceleration primarily originates from a source-drain voltage induced density gradient in the twodimensional electron gas (2DEG) in which polaritons are immersed. Remarkably, we show that the direction of the resulting force can be changed by an externally applied magnetic field, since the induced Hall voltage creates transverse density gradients. Finally, we extend this approach to demonstrate spin-selective acceleration of polaritons when the 2DEG is in the integer quantum Hall regime.

The sample used in this study is a monolithic distributed Bragg reflector optical microcavity with a quality factor $Q=5.5 \times 10^{3}$ grown by molecular-beam epitaxy. The structure contains a single optically active GaAs QW of $20 \mathrm{~nm}$ thickness located at the central antinode of the cavity field. This QW is remotely doped from both sides by $\mathrm{Si}$ impurities embedded in thin GaAs QWs (10 nm thickness) located at the nodes of the cavity field. The doping QWs thus provide electrons to the optically active QW where a 2DEG is formed with nominal electron density of $n_{e}=$ $0.33 \times 10^{11} \mathrm{~cm}^{-2}$ and mobility $\mu=1.6 \times 10^{6} \mathrm{~cm}^{2} \mathrm{~V}^{-1} \mathrm{~s}^{-1}$ (see Ref. [36] for further details on the sample fabrication). To study the interplay between polariton transport and 2DEG physics, the sample is etched in the form of a Hall bar with annealed electrical contacts to the 2DEG, as depicted in Figs. 1(a) and 1(b). A photoluminescence (PL) scan map of the sample, recorded at normal incidence (in-plane momentum $k_{\|}=0$ ), is shown in Fig. 1(d) outlining the shape of the Hall bar. The increase in emission energy of about $3 \mathrm{meV} / \mathrm{mm}$ from left to right is due to a (a)
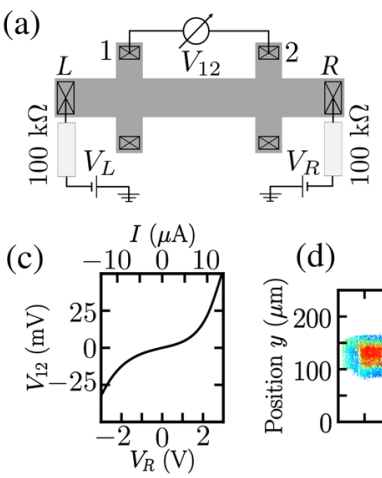

(b)

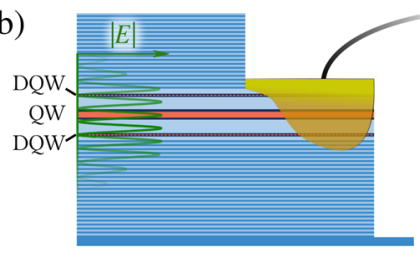

(d)

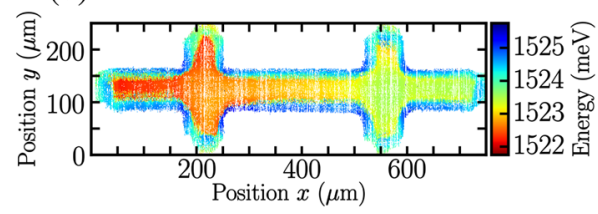

FIG. 1. Electrical and optical properties of the sample. (a) Layout of the Hall bar and the contacts. (b) Side view of the Hall bar. The top mirror has been etched to contact the 2DEG located in the center QW [doping quantum wells (DQW)]. (c) Current-voltage characteristic of the device where $V_{R}$ acts as current source and $V_{L}$ as drain. (d) Map of the lower polariton PL wavelength measured at normal incidence $\left(k_{\|}=0\right)$. wedge in the cavity length introduced during sample growth. The sample is cooled to a base temperature of $20 \mathrm{mK}$ in a dilution refrigerator with free space optical access enabling simultaneous position and momentumresolved microscopy, as well as magnetotransport experiments (see Supplemental Material [37]).

We first characterize the electrical properties of our sample by a four-point current-voltage $(I-V)$ measurement yielding the characteristic shown in Fig. 1(c). At low source-drain voltage bias the $I-V$ curve is linear with a 2 DEG sheet resistivity of $210 \Omega /$ sq. Increasing the sourcedrain bias to ca. $\pm 1 \mathrm{~V}$, the $I-V$ curve becomes nonlinear, entering a regime where the externally applied electrochemical potential leads to a spatially varying chemical potential across the 2DEG. At even larger source-drain bias, the 2DEG is depleted and we recover a linear $I-V$ characteristic with an increased resistivity of $1500 \Omega / \mathrm{sq}$, corresponding to electrical conduction in parallel channels (see Supplemental Material [37]).

These different regimes of the 2DEG behavior have a counterpart on the optical response of the system. Figure 2(a) shows the angular dispersion of the polaronpolariton states at zero source-drain bias. There, we identify four branches resulting from the hybridization of the cavity photon with the attractive and repulsive heavy-hole polarons and with the light-hole resonance. The dispersive polaron-polariton branches are well reproduced by the
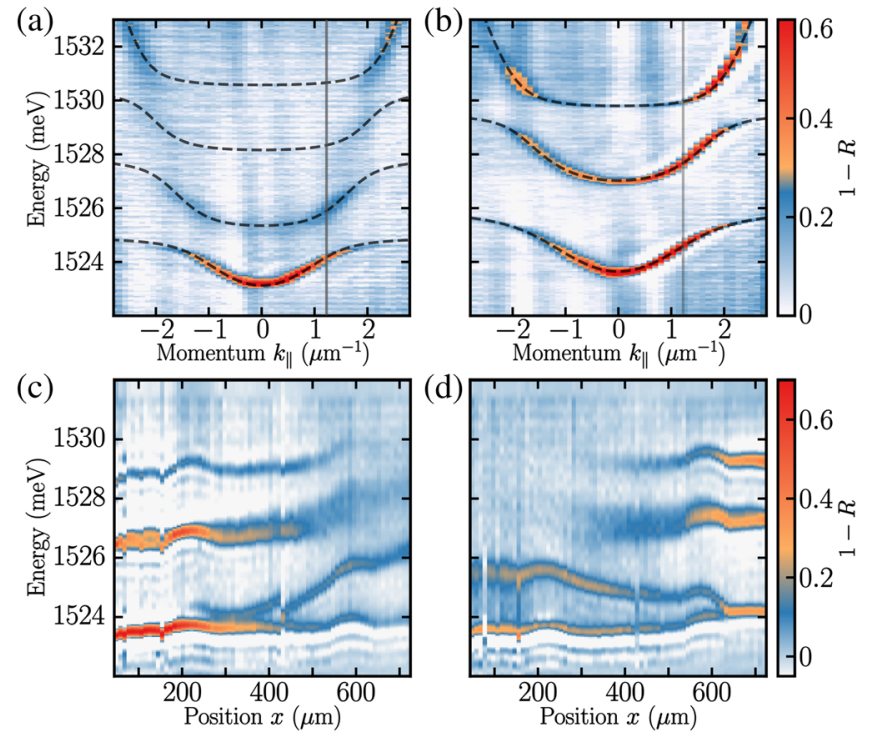

FIG. 2. Electrically controlled polariton landscape. (a) Normalized white-light reflectivity spectra showing the polaron-polariton dispersion at the nominal electron density. (b) Exciton-polariton dispersion in the depleted regime $\left(\Delta V_{L}=-6 \mathrm{~V}\right)$. Dashed lines are coupled oscillators fits to the data. (c),(d) Normalized white-light reflectivity spectra at $k_{\|}=$ $1.2 \mu \mathrm{m}^{-1}$ [vertical line in (a) and (b)] as a function of position with a negative bias voltage applied to the left contact $\left(\Delta V_{L}=-2.4 \mathrm{~V}\right)$ (c) and to the right contact $\left(\Delta V_{R}=-2.4 \mathrm{~V}\right)$ (d). 
coupled oscillators fit shown in dashed lines. Figure 2(b) shows the angular dispersion acquired while applying a large bias of $-6 \mathrm{~V}$ to the left contact and putting the right contact to ground, $\Delta V_{L}=-6 \mathrm{~V}$ [see Fig. 1(a)]. There, we measure the typical exciton-polariton dispersion of an intrinsic QW with anticrossings about the heavy-hole and light-hole exciton energies, as expected from the 2DEG depletion observed in the $I-V$ characteristic.

In between these two extremes, at intermediate bias voltages, the electron density shows smooth spatial gradients across the Hall bar as demonstrated in Fig. 2(c) for $\Delta V_{L}=-2.4 \mathrm{~V}$. Here, the polariton spectrum is recorded at positions across the long axis of the Hall bar and at fixed collection angle corresponding to $k_{\|}=1.2 \mu \mathrm{m}^{-1}$. On the left-hand side, where the negative bias is applied, the sample is devoid of electrons and the spectrum resembles a vertical cut in the dispersion of Fig. 2(b). Moving to the right, the electron density increases and we gradually recover polaron-polariton spectra corresponding to the dispersion of Fig. 2(a) as the oscillator strength is gradually transferred from the excitonic to the polaronic resonances. Reversing the applied electric field $\left(\Delta V_{R}=-2.4 \mathrm{~V}\right)$ inverts this density gradient, as shown in Fig. 2(d). Such electron density gradients constitute electrically tunable potential landscapes for polaritons which we explore in the following to transport neutral optical excitations.

To demonstrate polariton transport, we resonantly excite a polariton cloud and image its expansion in two opposing electron density gradients. The excitation laser, at $1524.0 \mathrm{meV}(813.54 \mathrm{~nm})$, is linearly polarized and focused in the central region of the Hall bar $(x=450 \mu$ m in Figs. 1 and 2), thus injecting a radially expanding cloud of polaritons with finite $k_{\|}$. The decaying polariton signal is collected by the same microscope objective lens and separated from the scattered excitation beam by polarization filtering. A finite strain along the crystalline axes in the structure allows us to obtain this resonance fluorescence (RF) signal by polarizing the excitation beam at $45^{\circ}$ with respect to the polarization eigenbasis defined by strain (see Supplemental Material [37]). Two images, $I_{R}$ and $I_{L}$, of this RF signal are acquired under source-drain biases of $\Delta V_{R}=-2.4 \mathrm{~V}$ and $\Delta V_{L}=-2.4 \mathrm{~V}$, respectively. These two voltages were chosen such that at the injection spot on the Hall bar, the electron densities are the same and the gradients are of opposite signs. This choice is not necessary but simplifies the observation of polariton acceleration, as there is no trivial difference between dispersions and thereby group velocities between the two images to be compared. Figure 3(a) shows the normalized difference of the two images, $\left(I_{R}-I_{L}\right) /\left(I_{R}+I_{L}\right)$, clearly demonstrating the ability to route polaritons by electrical means.

In order to model this effect, we measure the polariton dispersion at the two sides of the field of view $[x=0$ and $x=50 \mu \mathrm{m}$ in Fig. 3(a)] and perform a coupled oscillator fit. By linearly interpolating across the field of view, we
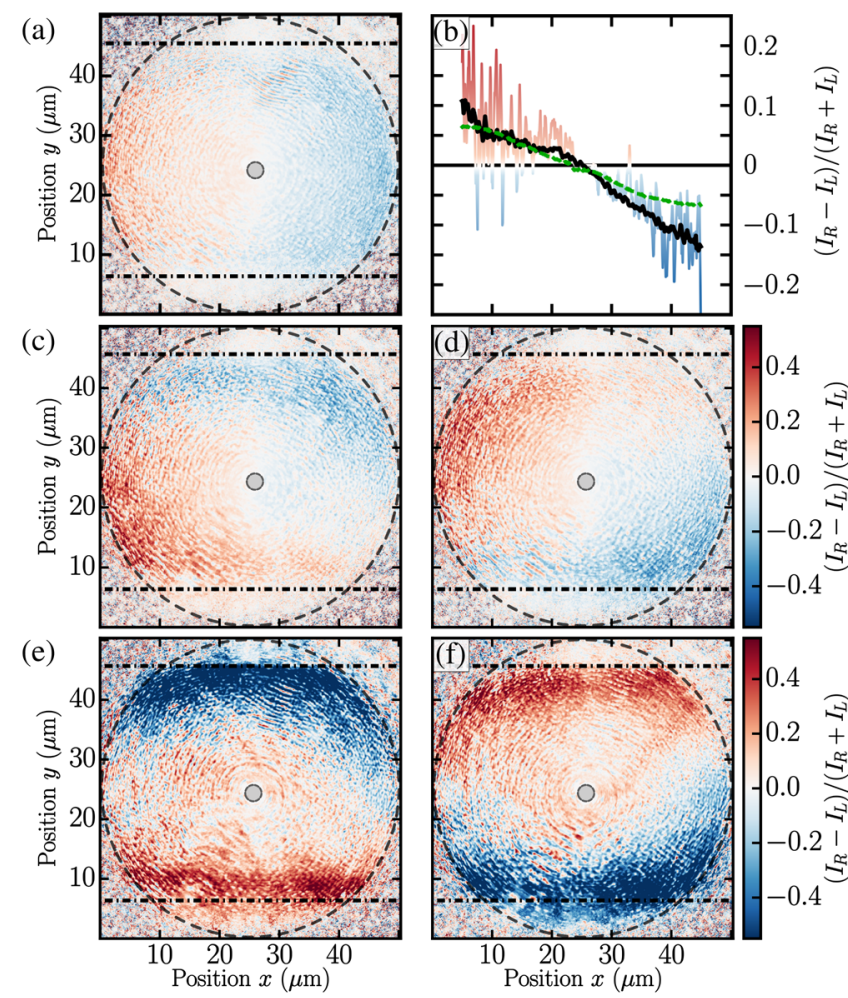

FIG. 3. Polariton acceleration in external electric and magnetic fields. (a) Normalized difference between two RF images of polariton flow in opposing electron density gradients. (b) Comparison with a trajectory-based model (dashed green line). The red and blue line is a line cut of (a) at $y=25 \mu \mathrm{m}$ and the black line is an average between $y=10$ and $y=40 \mu \mathrm{m}$. (c)-(f) Normalized difference between images $I_{R}, I_{L}$ as in (a) for magnetic fields: (c) $B=8 \mathrm{mT}$, (d) $B=-8 \mathrm{mT}$ (e), $B=40 \mathrm{mT}$, and (f) $B=$ $-40 \mathrm{mT}$. The horizontal dash-dotted lines in (a),(c)-(f) delimit the width of the Hall bar, the large circle is the field of view of the microscope, and the central gray disk is the excitation spot.

characterize the lower polariton energy landscape $E_{\mathrm{LP}}\left(x, k_{x}\right)$, separately for the two density gradients used to measure $I_{R}$ and $I_{L}$ (see Supplemental Material [37]). Starting from the classical Hamilton equations of motion in one dimension $(x)$, we propagate the experimentally determined initial conditions $k_{x}(0)= \pm 1.2 \mu \mathrm{m}^{-1}$ to predict the emission intensity $I(x)$, again repeated for the two energy landscapes corresponding to the two opposing gradients. As in the experiment, we calculate the normalized difference between the two cases and compare the result (green dashed line) with the experiment in Fig. 3(b). The red and blue colored line is a line cut through Fig. 3(a) at $y=25 \mu \mathrm{m}$ and the black curve is an average over $y$ between 10 and $40 \mu \mathrm{m}$. Remarkably, this simple approach allows us to obtain reasonable quantitative agreement with our data.

We can further control the polariton flow by applying a magnetic field perpendicular to the QW plane. In conjunction with a finite source-drain bias, this induces a Hall 
voltage transverse to the applied potential leading to charge redistribution in the $y$ direction $[42,43]$. The combined electric and magnetic fields now shape the electron density gradient which can be tuned in angle and magnitude, as demonstrated in Figs. 3(c)-3(f). Figure 3(c) corresponds to alternating voltage biases of $\Delta V_{R}=-2.4 \mathrm{~V}$ and $\Delta V_{L}=$ $-2.4 \mathrm{~V}$ and a fixed magnetic field of $8 \mathrm{mT}$ and shows the possibility to orient the polariton flow in a diagonal direction. Further increasing the magnetic field to $40 \mathrm{mT}$ [Fig. 3(e)] leads to polariton transport in the up-down direction with an intensity contrast of ca. 50\%. Moreover, flipping the sign of the applied magnetic field reverses the direction of polariton transport, as shown in Figs. 3(d) and $3(\mathrm{f})$.

While these results demonstrate the possibility to transport dressed photons (i.e., polaritons) by electric and magnetic fields, it should be noted that we do not observe here a Lorentz force for photons. In particular, the force acting on polaritons does not appear to depend on the direction of their motion. As clearly shown in Figs. 3(e) and 3(f), both the polaritons propagating to the left and to the right are deflected in the same direction. The polariton acceleration is determined only by the electron density gradient which in turn is controlled by the combination of magnetic field and electrical bias. In other words, the nonperturbative coupling of polaritons to itinerant electrons allows for the control of photons by electromagnetic forces acting on the electronic sector. In the last part of this article, we demonstrate how this idea can be extended to realize transverse polariton spin currents reminiscent of an intrinsic spin-Hall effect when the 2DEG is close to the $\nu=1$ integer quantum Hall state.

At low temperature and under a strong magnetic field, the optical excitation spectrum of a high-mobility 2DEG exhibits energy gaps due to the quantization of cyclotron orbits. The corresponding Landau levels are further split in energy by a Zeeman field, forming a ladder of spin subbands for the electrons. As the occupancy of this ladder is varied (e.g., by tuning the magnetic field), the $2 \mathrm{DEG}$ undergoes phase transitions between electronic ground states of different spin polarization $S_{z}$. In particular, when the lower spin subband of the first Landau level is completely filled $(\nu=1)$, the electronic ground state is an itinerant ferromagnet with strong spin polarization. Remarkably, the fact that polaron dressing occurs exclusively in the configuration where the optically excited electron and the electrons in the 2DEG dressing cloud have opposite spin renders the polaron very sensitive to the spin polarization of the $2 \mathrm{DEG}$. At $\nu=1$, the polaron oscillator strength is maximum for right-hand circularly polarized light $\sigma^{+}$since most of the 2DEG electrons are in the spinup state, thus allowing for efficient dressing. Conversely, the oscillator strength is reduced for left-hand circularly polarized light $\sigma^{-}$due to the absence of spin-down electrons. The ensuing variations of polaron oscillator strength have a direct counterpart in the Rabi splittings $\Omega_{\sigma^{ \pm}}$of the corresponding polaron-polariton branches, as shown in Fig. 4(a) and investigated in detail in Refs. [36,44]. The degree of electron spin polarization can be inferred from the Rabi splittings as $S_{z} \simeq\left(\Omega_{\sigma^{+}}^{2}-\right.$ $\left.\Omega_{\sigma^{-}}^{2}\right) /\left(\Omega_{\sigma^{+}}^{2}+\Omega_{\sigma^{-}}^{2}\right) \simeq 70 \%$ at $\nu=1 \quad(1.26 \mathrm{~T})$. In this
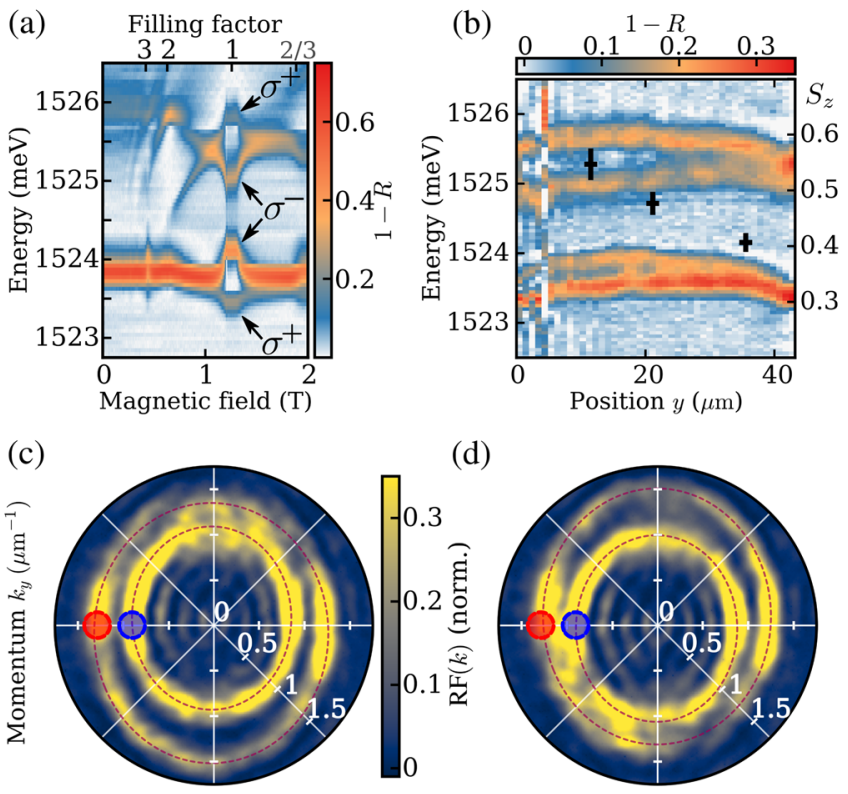

(d)
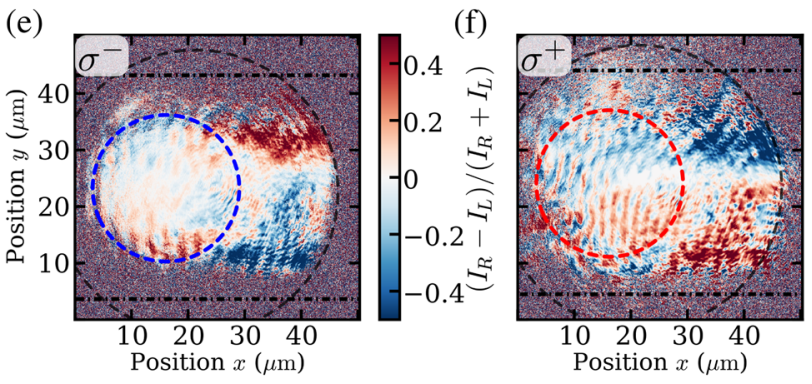

FIG. 4. Spin-selective polariton acceleration. (a) Normalized white-light reflectivity spectra measured at $k_{\|}=1.2 \mu \mathrm{m}^{-1}$ as a function of magnetic field. The corresponding Landau level filling factor is determined from an independent magnetotransport measurement. (b) Normalized reflectivity spectra at $k_{\|}=$ $1.2 \mu \mathrm{m}^{-1}$ and $B=1.1 \mathrm{~T}$ across the vertical $y$ direction on the Hall bar, with a source-drain bias $\Delta V_{R}=-0.17 \mathrm{~V}$. Black points indicate the electron spin polarization $S_{z}$ measured at three different positions. (c),(d) Momentum-resolved polariton RF emission under cross-linear polarization at $1523.8 \mathrm{meV}$ (813.62 nm) for (c) $\Delta V_{R}=-0.17 \mathrm{~V}$ and (d) $\Delta V_{L}=-0.35 \mathrm{~V}$. The red dashed lines are guides to the eye. (e),(f) Normalized difference between two RF images of right-propagating polaritons acquired with $\Delta V_{R}=-0.17 \mathrm{~V}$ and $\Delta V_{L}=-0.35 \mathrm{~V}$. (e) Excitation of $\sigma^{-}$polaritons at $k_{x}=-0.9 \mu \mathrm{m}^{-1}, k_{y}=0$. (f) Excitation of $\sigma^{+}$polaritons at $k_{x}=-1.3 \mu \mathrm{m}^{-1}, k_{y}=0$. The horizontal dash-dotted lines delimit the width of the Hall bar, the large circle is the field of view of the microscope. The red and blue rings in (c)-(f) show the excitation spots in real and momentum spaces for $\sigma^{+}$and $\sigma^{-}$polaritons, respectively. 
quantum Hall regime, the charge-density gradients demonstrated above translate into gradients of 2DEG spin polarization, resulting in optical spin-contrasted forces for polaritons.

Figure 4(b) shows the evolution of the polariton spectrum at $k_{\|}=1.2 \mu \mathrm{m}^{-1}$, recorded from the lower edge to the upper edge of the Hall bar ( $y$ direction) under a voltage bias of $\Delta V_{R}=-0.17 \mathrm{~V}$ and a magnetic field of $1.1 \mathrm{~T}$. We observe four energy branches, as expected from a vertical cut in Fig. 4(a) near $\nu=1$, where the two inner branches are the $\sigma^{-}$-polarized lower and upper polaritons and the two outer ones are the $\sigma^{+}$-polarized lower and upper polaritons. In order to calculate the degree of spin polarization, we acquired energy dispersions at three different $y$ positions and extracted the Rabi splittings by a fit based on coupled oscillators (see Ref. [37]). As can be seen in Fig. 4(b), the degree of electron spin polarization of the 2DEG (black marks, right axis) evolves across the Hall bar and can be controlled electrically as demonstrated in the Supplemental Material [37]. Remarkably, such variations in $S_{z}$ constitute gradients of opposite signs for the $\sigma^{+}$and $\sigma^{-}$polaronpolariton energy landscapes.

To investigate the resulting spin-dependent polariton acceleration, we first perform momentum-resolved measurements by imaging the polariton RF emission (see Supplemental Material [37]). An excitation energy of $1523.8 \mathrm{meV}(813.62 \mathrm{~nm})$ is chosen to intercept both $\sigma^{+}$ and $\sigma^{-}$lower polariton dispersions at finite $k_{\|}$. Figures 4(c) and 4(d) show the momentum-resolved polariton RF signal for $\Delta V_{R}=-0.17 \mathrm{~V}$ and $\Delta V_{L}=-0.35 \mathrm{~V}$, respectively. As can be seen in Fig. 4(c), the inner $\sigma^{-}$-polarized branch is shifted toward positive $k_{y}$ while the outer $\sigma^{+}$-polarized branch is shifted toward negative $k_{y}$. This observation directly demonstrates an in-plane acceleration whose sign depends on the spin of the polaritons $S_{z}^{\mathrm{pol}}$ in the given quantum state [45]. The effect is reversed in Fig. 4(d), where the external bias and thereby the gradient in electron spin polarization points in the opposite $y$ direction. The real-space counterpart of this acceleration allows for the generation of transverse optical spin currents, reminiscent of an intrinsic optical spin-Hall effect [46-52].

To demonstrate the generation of transverse polariton spin currents, we inject polariton waves of well-defined momenta by focusing the excitation beam in the back-focal plane of the objective lens. Figures 4(e) and 4(f) correspond to excitation at $k_{x}=-0.9 \mu \mathrm{m}^{-1}, k_{y}=0$ and $k_{x}=-1.3 \mu \mathrm{m}^{-1}$, $k_{y}=0$, resulting in right propagating polaritons with $\sigma^{-}$ and $\sigma^{+}$polarization, respectively. The normalized difference of the propagation images, acquired with the two different voltage biases, indeed reveals opposite acceleration of $\sigma^{-}$and $\sigma^{+}$polaritons along the $y$ direction.

The spin-dependent momentum shifts demonstrated here, although capable of generating transverse spin currents, remain fundamentally different from the usual
Rashba-type coupling at the origin of standard spin-Hall effects. Instead, the interaction reported here is analogous to a force for photons, where the spatially varying electron spin polarization $\left(S_{z}\right)$ acts as an accelerating potential, sorting polaritons of different spin $\left(S_{z}^{\mathrm{pol}}\right)$ in different directions:

$$
\mathbf{F}_{\text {photon }} \sim S_{z}^{\text {pol }} \nabla S_{z}
$$

It should be noted that the evolution of electron spin polarization around $\nu=1$ quantum Hall plateau is widely believed to involve the proliferation of skyrmions in the quantum Hall ferromagnetic state due to the interplay between Zeeman and Coulomb energies [53]. The spinsinglet polaron-polariton dressing thus constitutes a new interface for coupling the optical spin of photons to the electronic spin excitations of 2DEGs. The behavior of such interactions in the fractional quantum Hall regime where excitons may be dressed by fractionally charged quasiparticles as well as the residual interactions between these fractional quantum Hall polaritons remain to be explored [54].

In summary, we demonstrated novel ways of controlling photons with external electric and magnetic fields, which are enabled by their hybridization with polarization waves in a medium. While these results were obtained in the context of exciton-polaritons interacting with electrons, we highlight that the underlying mechanism is general and could allow for the electrical control of photons hybridized with other kinds of polarization waves such as phonons or plasmons, provided that the quanta of polarization concurrently interact with free electrons or holes.

A nonequilibrium electron density gradient is shown to act as an effective electric field for polaron-polaritons which is tunable in strength and direction. We foresee that this effective electric field could be further controlled by tailoring the 2DEG density, e.g., using patterned electrodes. By mapping the energy landscape of the lower polariton, we reach quantitative agreement between a simple trajectory-based model and the observed polariton acceleration. Our experiment constitutes an alternative to the already proposed polariton drag effect for exerting electromagnetic forces on neutral optical excitations [18,35]. We emphasize that the electron density gradients we exploit are generic for low-density 2DEGs when large source-drain voltages are applied and therefore need to be considered in view of polariton drag experiments.

In the integer quantum Hall regime, we demonstrated that electron spin depolarization, induced by the proliferation of skyrmions as the electron density gradient pushes the system away from $\nu=1$ filling, constitutes a scalar potential for the optical spin of photons and results in spindependent accelerations. This observation opens a new interface between optical and electronic spin sectors as 
magnetic excitations of the 2DEG, such as magnons, could be used for spin-selective drag of photons.

The data that support the findings of this article are available in the ETH Research Collection [55].

\section{ACKNOWLEDGMENTS}

We thank H.-T. Lim, S. Ravets, E. Togan, and Y. Tsuchimoto for extensive help with the design and fabrication of the sample. We also acknowledge fruitful discussions with O. Cotlet, E. Demler, T. Ihn, P. Märki, A. Popert, R. Schmidt, Y. Shimazaki, and T. Smolenski. This work was supported by the Swiss National Science Foundation (NCCR Quantum Science and Technology). This project has received funding from the European Research Council under the Grant Agreement No. 671000 (POLTDES).

[1] L. Lu, J. D. Joannopoulos, and M. Soljačić, Topological Photonics, Nat. Photonics 8, 821 (2014).

[2] T. Ozawa, H. M. Price, A. Amo, N. Goldman, M. Hafezi, L. Lu, M. C. Rechtsman, D. Schuster, J. Simon, O. Zilberberg, and I. Carusotto, Topological Photonics, Rev. Mod. Phys. 91, 015006 (2019).

[3] M. Aidelsburger, S. Nascimbene, and N. Goldman, Artificial Gauge Fields in Materials and Engineered Systems, C. R. Phys. Quantum Simul. 19, 394 (2018).

[4] I. Carusotto and C. Ciuti, Quantum Fluids of Light, Rev. Mod. Phys. 85, 299 (2013).

[5] J. L. O'Brien, A. Furusawa, and J. Vučković, Photonic Quantum Technologies, Nat. Photonics 3, 687 (2009).

[6] D. Sanvitto and S. Kéna-Cohen, The Road towards Polaritonic Devices, Nat. Mater. 15, 1061 (2016).

[7] C. Schneider, K. Winkler, M. D. Fraser, M. Kamp, Y. Yamamoto, E. A. Ostrovskaya, and S. Höfling, ExcitonPolariton Trapping and Potential Landscape Engineering, Rep. Prog. Phys. 80, 016503 (2016).

[8] N. Engheta, Circuits with Light at Nanoscales: Optical Nanocircuits Inspired by Metamaterials, Science 317, 1698 (2007).

[9] H.-T. Lim, E. Togan, M. Kroner, J. Miguel-Sanchez, and A. Imamoğlu, Electrically Tunable Artificial Gauge Potential for Polaritons, Nat. Commun. 8, 1 (2017).

[10] T. Karzig, C.-E. Bardyn, N. H. Lindner, and G. Refael, Topological Polaritons, Phys. Rev. X 5, 031001 (2015).

[11] O. Bleu, D. D. Solnyshkov, and G. Malpuech, Measuring the Quantum Geometric Tensor in Two-Dimensional Photonic and Exciton-Polariton Systems, Phys. Rev. B 97, 195422 (2018).

[12] A. Gutiérrez-Rubio, L. Chirolli, L. Martín-Moreno, F. J. García-Vidal, and F. Guinea, Polariton Anomalous Hall Effect in Transition-Metal Dichalcogenides, Phys. Rev. Lett. 121, 137402 (2018).

[13] S. Klembt, T. H. Harder, O. A. Egorov, K. Winkler, R. Ge, M. A. Bandres, M. Emmerling, L. Worschech, T. C. H. Liew, M. Segev, C. Schneider, and S. Höfling,
Exciton-Polariton Topological Insulator, Nature (London) 562, 552 (2018).

[14] D. Sanvitto, F. Pulizzi, A. J. Shields, P. C. M. Christianen, S. N. Holmes, M. Y. Simmons, D. A. Ritchie, J. C. Maan, and M. Pepper, Observation of Charge Transport by Negatively Charged Excitons, Science 294, 837 (2001).

[15] F. Pulizzi, D. Sanvitto, P. C. M. Christianen, A. J. Shields, S. N. Holmes, M. Y. Simmons, D. A. Ritchie, M. Pepper, and J. C. Maan, Optical Imaging of Trion Diffusion and Drift in GaAs Quantum Wells, Phys. Rev. B 68, 205304 (2003).

[16] D. V. Kulakovskii and Y. E. Lozovik, Screening and Rearrangement of Excitonic States in Double Layer Systems, J. Exp. Theor. Phys. 98, 1205 (2004).

[17] O. L. Berman, R. Y. Kezerashvili, and Y. E. Lozovik, Can We Move Photons?, Phys. Lett. A 374, 3681 (2010).

[18] O. L. Berman, R. Y. Kezerashvili, and Y. E. Lozovik, Drag Effects in a System of Electrons and Microcavity Polaritons, Phys. Rev. B 82, 125307 (2010).

[19] B. N. Narozhny and A. Levchenko, Coulomb Drag, Rev. Mod. Phys. 88, 025003 (2016).

[20] D. M. Myers, B. Ozden, J. Beaumariage, L. N. Pfeiffer, K. West, and D. W. Snoke, Pushing Photons with Electrons: Observation of the Polariton Drag Effect, arXiv:1808 .07866 .

[21] I. Y. Chestnov, Y. G. Rubo, and A. V. Kavokin, PseudoDrag of a Polariton Superfluid, Phys. Rev. B 100, 085302 (2019).

[22] G. Malpuech, A. Kavokin, A. Di Carlo, and J. J. Baumberg, Polariton Lasing by Exciton-Electron Scattering in Semiconductor Microcavities, Phys. Rev. B 65, 153310 (2002).

[23] P. G. Lagoudakis, M. D. Martin, J. J. Baumberg, A. Qarry, E. Cohen, and L. N. Pfeiffer, Electron-Polariton Scattering in Semiconductor Microcavities, Phys. Rev. Lett. 90, 206401 (2003).

[24] A. Qarry, G. Ramon, R. Rapaport, E. Cohen, A. Ron, A. Mann, E. Linder, and L. N. Pfeiffer, Nonlinear Emission due to Electron-Polariton Scattering in a Semiconductor Microcavity, Phys. Rev. B 67, 115320 (2003).

[25] M. Perrin, P. Senellart, A. Lemaître, and J. Bloch, Polariton Relaxation in Semiconductor Microcavities: Efficiency of Electron-Polariton Scattering, Phys. Rev. B 72, 075340 (2005).

[26] T. Brunhes, R. André, A. Arnoult, J. Cibert, and A. Wasiela, Oscillator Strength Transfer from $X$ to $X+$ in a CdTe Quantum-Well Microcavity, Phys. Rev. B 60, 11568 (1999).

[27] R. Rapaport, R. Harel, E. Cohen, A. Ron, E. Linder, and L. N. Pfeiffer, Negatively Charged Quantum Well Polaritons in a GaAs/AlAs Microcavity: An Analog of Atoms in a Cavity, Phys. Rev. Lett. 84, 1607 (2000).

[28] R. Rapaport, E. Cohen, A. Ron, E. Linder, and L. N. Pfeiffer, Negatively Charged Polaritons in a Semiconductor Microcavity, Phys. Rev. B 63, 235310 (2001).

[29] R. A. Suris, in Optical Properties of 2D Systems with Interacting Electrons, NATO Science Series, edited by W. J. Ossau and R. Suris (Springer, Dordrecht, 2003) pp. 111-124.

[30] M. Combescot and J. Tribollet, Trion Oscillator Strength, Solid State Commun. 128, 273 (2003).

[31] M. Combescot, J. Tribollet, G. Karczewski, F. Bernardot, C. Testelin, and M. Chamarro, Many-Body Origin of the Trion 
Line in Doped Quantum Wells, Europhys. Lett. 71, 431 (2005).

[32] M. Sidler, P. Back, O. Cotlet, A. Srivastava, T. Fink, M. Kroner, E. Demler, and A. Imamoglu, Fermi PolaronPolaritons in Charge-Tunable Atomically Thin Semiconductors, Nat. Phys. 13, 255 (2017).

[33] D. K. Efimkin and A. H. MacDonald, Many-Body Theory of Trion Absorption Features in Two-Dimensional Semiconductors, Phys. Rev. B 95, 035417 (2017).

[34] D. K. Efimkin and A. H. MacDonald, Exciton-Polarons in Doped Semiconductors in a Strong Magnetic Field, Phys. Rev. B 97, 235432 (2018).

[35] O. Cotleţ, F. Pientka, R. Schmidt, G. Zarand, E. Demler, and A. Imamoglu, Transport of Neutral Optical Excitations Using Electric Fields, Phys. Rev. X 9, 041019 (2019).

[36] S. Ravets, P. Knüppel, S. Faelt, O. Cotlet, M. Kroner, W. Wegscheider, and A. Imamoglu, Polaron Polaritons in the Integer and Fractional Quantum Hall Regimes, Phys. Rev. Lett. 120, 057401 (2018).

[37] See Supplemental Material at http://link.aps.org/ supplemental/10.1103/PhysRevX.10.011040 for additional information about the optical setup, electrical and polarization properties of the sample, the theoretical model, and spin density gradients, which includes Refs. [38-41].

[38] D. Fritzsche, Heterostructures in MODFETs, Solid-State Electron. 30, 1183 (1987).

[39] M. J. Kane, N. Apsley, D. A. Anderson, L. L. Taylor, and T. Kerr, Parallel Conduction in GaAs $/ \mathrm{Al}_{x} \mathrm{Ga}_{1-x} \mathrm{As}$ Modulation Doped Heterojunctions, J. Phys. C 18, 5629 (1985).

[40] M. Reed, W. Kirk, and P. Kobiela, Investigation of Parallel Conduction in $\mathrm{GaAs} / \mathrm{Al}_{x} \mathrm{Ga}_{1-x} \mathrm{As}$ Modulation-Doped Structures in the Quantum Limit, IEEE J. Quantum Electron. 22, 1753 (1986).

[41] M. Steger, G. Liu, B. Nelsen, C. Gautham, D. W. Snoke, R. Balili, L. Pfeiffer, and K. West, Long-Range Ballistic Motion and Coherent Flow of Long-Lifetime Polaritons, Phys. Rev. B 88, 235314 (2013).

[42] P. F. Fontein, J. A. Kleinen, P. Hendriks, F. A. P. Blom, J. H. Wolter, H. G. M. Lochs, F. A. J. M. Driessen, L. J. Giling, and C. W. J. Beenakker, Spatial Potential Distribution in GaAs/ $/ \mathrm{Al}_{x} \mathrm{Ga}_{1-x} \mathrm{As}$ Heterostructures under Quantum Hall Conditions Studied with the Linear Electro-optic Effect, Phys. Rev. B 43, 12090 (1991).

[43] M. Cage and C. Lavine, Potential and Current Distributions Calculated Across A Quantum Hall-Effect Sample at Low and High Currents, J. Res. Natl. Inst. Stand. Technol. 100, 529 (1995).

[44] S. Smolka, W. Wuester, F. Haupt, S. Faelt, W. Wegscheider, and A. Imamoglu, Cavity Quantum Electrodynamics with Many-Body States of a Two-Dimensional Electron Gas, Science 346, 332 (2014).

[45] K. V. Kavokin, I. A. Shelykh, A. V. Kavokin, G. Malpuech, and P. Bigenwald, Quantum Theory of Spin Dynamics of Exciton-Polaritons in Microcavities, Phys. Rev. Lett. 92, 017401 (2004).

[46] M. Onoda, S. Murakami, and N. Nagaosa, Hall Effect of Light, Phys. Rev. Lett. 93, 083901 (2004).

[47] A. Kavokin, G. Malpuech, and M. Glazov, Optical Spin Hall Effect, Phys. Rev. Lett. 95, 136601 (2005).

[48] C. Leyder, M. Romanelli, J. P. Karr, E. Giacobino, T. C. H. Liew, M. M. Glazov, A. V. Kavokin, G. Malpuech, and A. Bramati, Observation of the Optical Spin Hall Effect, Nat. Phys. 3, 628 (2007).

[49] A. Amo, T. C. H. Liew, C. Adrados, E. Giacobino, A. V. Kavokin, and A. Bramati, Anisotropic Optical Spin Hall Effect in Semiconductor Microcavities, Phys. Rev. B 80, 165325 (2009).

[50] M. Maragkou, C. E. Richards, T. Ostatnický, A. J. D. Grundy, J. Zajac, M. Hugues, W. Langbein, and P. G. Lagoudakis, Optical Analogue of the Spin Hall Effect in a Photonic Cavity, Opt. Lett. 36, 1095 (2011).

[51] K. Lekenta, M. Król, R. Mirek, K. Łempicka, D. Stephan, R. Mazur, P. Morawiak, P. Kula, W. Piecek, P. G. Lagoudakis, B. Piętka, and J. Szczytko, Tunable Optical Spin Hall Effect in a Liquid Crystal Microcavity, Light Sci. Appl. 7, 1 (2018).

[52] A. Gianfrate, O. Bleu, L. Dominici, V. Ardizzone, M. De Giorgi, D. Ballarini, K. West, L. N. Pfeiffer, D. D. Solnyshkov, D. Sanvitto, and G. Malpuech, Direct Measurement of the Quantum Geometric Tensor in a TwoDimensional Continuous Medium, arXiv:1901.03219.

[53] S. M. Girvin, Spin and Isospin: Exotic Order in Quantum Hall Ferromagnets, in The Multifaceted Skyrmion, edited by M. Rho and I. Zahed (World Scientific, Singapore, 2015), pp. 351-365.

[54] P. Knüppel, S. Ravets, M. Kroner, S. Fält, W. Wegscheider, and A. Imamoglu, Nonlinear Optics in the Fractional Quantum Hall Regime, Nature (London) 572, 91 (2019).

[55] P. Knüppel, ETH Zurich Research Collection, http://hdl .handle.net/20.500.11850/387816 (2020). 